\title{
LETTERS
}

\section{Single mineral particle makes an electron point source}

\author{
Evelyne Salançon \\ CNRS, Aix-Marseille Université, CINaM UMR 7325, Campus de Luminy, Case 913, 13288 Marseille Cedex 9, \\ France \\ Rachid Daineche \\ CNRS, Aix-Marseille Université, IM2NP UMR 7334, Faculté des Sciences et Techniques, \\ Avenue Escadrille Normandie Niemen, Case 142, 13397 Marseille Cedex 20, France \\ Olivier Grauby and Roger Morin ${ }^{\text {a) }}$ \\ CNRS, Aix-Marseille Université, CINaM UMR 7325, Campus de Luminy, Case 913, 13288 Marseille Cedex 9, \\ France
}

(Received 7 November 2014; accepted 16 March 2015; published 27 March 2015)

\begin{abstract}
A mineral (celadonite, kaolinite) nanometer-thick particle deposited on a flat carbon film or at the apex of a carbon fiber provides electron emission at low applied fields. Voltage and time dependences of the emission intensity are studied, and a model of the underlying mechanism is proposed. An electron point source providing emission from a single particle is built and characterized. (C) 2015 American Vacuum Society. [http://dx.doi.org/10.1116/1.4916237]
\end{abstract}

\section{INTRODUCTION}

Electron field emission at room temperature at low applied macroscopic fields ${ }^{1}$ (approximately a few $\mathrm{V} / \mu \mathrm{m}$ ) is reported by many authors. ${ }^{2-12}$ But, since the work function of a material in vacuum is always higher than $1.5 \mathrm{eV}$ ( $\mathrm{Cs}$ on $\mathrm{W}$, for instance $^{13}$ ), field emission current density is negligible for local fields smaller than a fraction of $\mathrm{V} / \mathrm{nm}$. This means that the reported low macroscopic field values are at least two orders of magnitude smaller than the local field values required both by theory and by well-defined field emission experiments performed on metallic field emitters. ${ }^{14-16}$ However, because the local field that governs field emission is the field in vacuum up to only a few nanometers from the material vacuum interface, it is complicated to measure. First, because even in the simple case of conductors and with the exception of plane capacitor electrode geometry, this local field is determined from a spatially inhomogeneous field distribution requiring knowledge of electrode geometry from macroscopic to nanometric scales. ${ }^{17}$ Second, as insulators are often present in low macroscopic-field emission experiments, any charges present on or in the insulators contribute to the local field, making it essential to know the charge distribution in order to determine the local field. Yet despite these difficulties and requirements, electron emission at low applied macroscopic fields from various cathodes covered by thin films of material like diamond, carbon, various oxides, polymers, and composite films are reported. ${ }^{18,19}$ A very convincing theoretical and experimental case based on injection of hot electrons into insulating particles on metal is made by Bayliss and Latham ${ }^{20}$ to explain apparent electron field emission at low fields. An overview of the various approaches is given by Forbes. ${ }^{1}$

a)Electronic mail: morin@ cinam.univ-mrs.fr
In two previous publications, we report on electron emission from films obtained by liquid drop deposition of mineral particles on carbon membranes, ${ }^{21}$ and on the coherence of the electron beam emitted by a single particle of such films. ${ }^{22}$ Here, we report the measurement of electron emission from a single mineral particle deposited either on a flat carbon film or at the apex of a carbon fiber and the building of a source providing emission from a single particle. We also propose a mechanism explaining the low-macroscopic-field emission.

\section{EXPERIMENTAL, RESULTS, AND DISCUSSION}

\section{A. Particle deposition}

Celadonite and kaolinite are metal phyllosilicate minerals. Most experiments were performed with celadonite, which belongs to the mica group, well known for its insulating behavior. $^{23,24}$ This behavior is visible in scanning electron microscopy (SEM) by some contrast change with imaging voltage. The precise chemical composition of celadonite samples we use is $\mathrm{Si}_{3.87} \mathrm{Al}_{0.13} \mathrm{O}_{10}(\mathrm{OH})_{2} \mathrm{Al}_{0.38} \mathrm{Fe}_{0.87} \mathrm{Mg}_{0.67} \mathrm{~K}$. We prepare solutions containing mineral particles by first pouring celadonite or kaolinite powder (from as much as $1 \mathrm{mg}$ down to $1 \mu \mathrm{g}$ ) into $7 \mathrm{ml}$ of pure water. Ultrasounds are then applied to the solutions to destroy particle agglomerates and to disperse particles within the water.

For experiments on carbon films, using a micropipette, a droplet of $7 \mu \mathrm{l}$ is deposited onto the carbon film fixed on a $3.1 \mathrm{~mm}$ diameter electron microscopy grid. ${ }^{25}$ Using this procedure, transmission electron microscopy observation shows that particle density as low as $10^{9} / \mathrm{m}^{2}$ can be obtained (the particle density is measured from the number of particles sized over $1 \mu \mathrm{m}$ inside an area of $100 \mu \mathrm{m} \times 100 \mu \mathrm{m} / \mathrm{using}$ software "IMAGEJ"). This low density is required to select the current emitted by a single particle and corresponds to about $30 \mu \mathrm{m}$ distance between particles. 
For experiments on a carbon fiber, the challenge is to obtain only one particle deposited at the apex of the $12 \mu \mathrm{m}$ diameter fiber in order to get electron emission solely from this particle, since the field is higher at the apex of the carbon fiber. This is successfully obtained by quickly plunging into, and removing from the apex of the carbon fiber, a droplet of the solution containing mineral particles. For ease of manipulation, the base of the fiber is fixed inside a $90 \mu \mathrm{m}$ diameter stainless steel tube. The deposition is then performed under an optical microscope $(\times 5)$ by approaching the droplet formed at the apex of a micropipette from the apex of the fiber. SEM is used to observe the result of the deposition procedure.

Results on these depositions of celadonite particles on a carbon film and on the apex of a carbon fiber are shown in Fig. 1. Statistical analysis of electron micrographs of celadonite particles shows that the average width of particles is $1 \mu \mathrm{m}$, and that the average length to width ratio is 2.5 . Atomic force microscope observations give the same results, with a thickness of less than $75 \mathrm{~nm}$ for more than half the particles. Similar results are observed for kaolinite, which can be even less thick, measuring as little as $60 \mathrm{~nm}$.

\section{B. Electron emission}

Different electrical arrangements are required for film and fiber experiments, since many particles emit for a film whereas a single particle emits for a well-prepared fiber.

For films, an electron microscopy copper grid $(3.1 \mathrm{~mm}$ in diameter, 300 meshes) is placed $300 \mu \mathrm{m}$ parallel to the grid supporting the carbon membrane using mica spacers and a dedicated holder. This holder is placed inside an ultra high vacuum chamber $10 \mathrm{~mm}$ in front of a $100 \mu \mathrm{m}$ diameter metal diaphragm. Projected images of this diaphragm illuminated by electron beams emitted by each particle are formed on a fluorescent screen placed $564 \mathrm{~mm}$ from the diaphragm [Figs. 2(a) and 2(b)].

By decreasing the particle density to $109 / \mathrm{m}^{2}$, and moving the source relative to the diaphragm, we eventually end up with one single image of this diaphragm [Fig. 2(b)]. It thus becomes possible to isolate current I emitted from a single particle in the solid angle covered by the diaphragm from the source $\left(7.4 \times 10^{-5} \mathrm{sr}\right)$. Using a Keithley 485 picoammeter, $\mathrm{I}$ is then measured versus voltage $\mathrm{V}$ applied between the film and the grid. The applied field is the voltage divided by the distance between the film and the grid $(300 \mu \mathrm{m})$. I vs
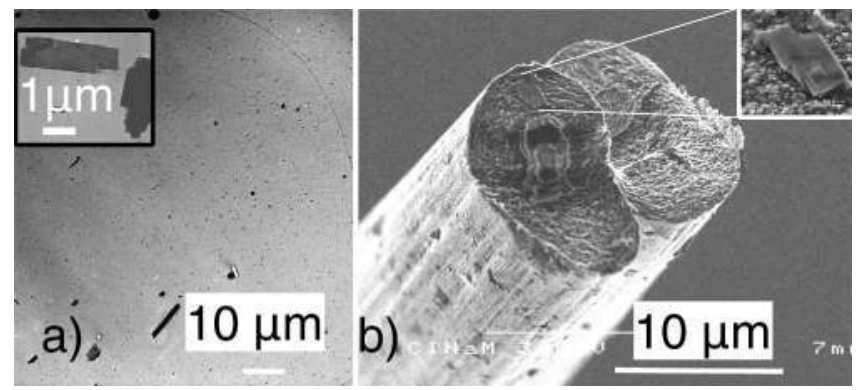

FIG. 1. Transmission (a) and scanning (b) electron micrographs of a celadonite particle on a carbon film (a) and on a carbon fiber (b). Insets show higher magnification micrographs.

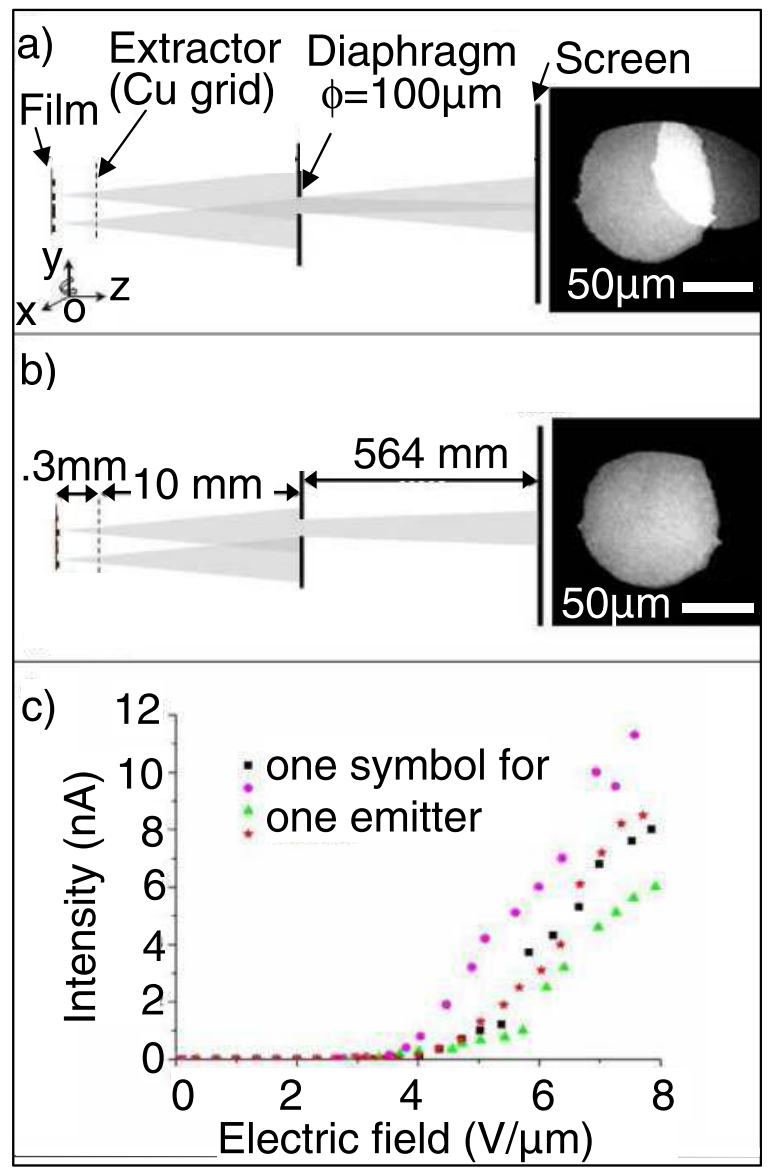

FIG. 2. (Color online) Electron projection image of a $100 \mu \mathrm{m}$ diameter metal diaphragm: (a) illuminated by two beams originating from two sources and (b) illuminated by a beam originating from a single source. (c) Current I vs field (ratio of voltage to film-grid distance) of electron emission from four single celadonite particles in the solid angle $\left(7.4 \times 10^{-5} \mathrm{sr}\right)$ covered by the diaphragm from the emitting particle.

$\mathrm{V}$ characteristics obtained thereby on four different single celadonite particles are shown in Fig. 2(c).

For a fiber experiment, the fiber and the extraction grid can be moved using a mechanical micromanipulator. We check that carbon fibers free from mineral particles do emit but at a much higher voltage $(>5 \mathrm{kV})$ than when mineral particles are present $(<1 \mathrm{kV})$. The extraction grid supports a lacey carbon membrane, ${ }^{25}$ and a finely defined projection image of this membrane ${ }^{26}$ is observable on a fluorescent screen placed $70 \mathrm{~cm}$ from the source [Fig. 3(a)]. Lensless projection imaging capability illustrated by Figs. 2(a) and 3(a) is a direct proof of the point source character of the emission. Note that images of thin filaments of the membrane are not simple shadows but inline holograms of these filaments relying on the coherent illumination provided by the small size of the source..$^{22,27,28}$

Because an actual projection image of an object is the convolution of the perfect point projection image of this object by that one of the actual source, an upper value of the virtual size of the source is obtained from the best resolved detail in the projection images. This value is clearly [from Fig. 3(a)] below $\sim 10 \mathrm{~nm}$, and much smaller than the size of a celadonite particle. Therefore, current densities can be 


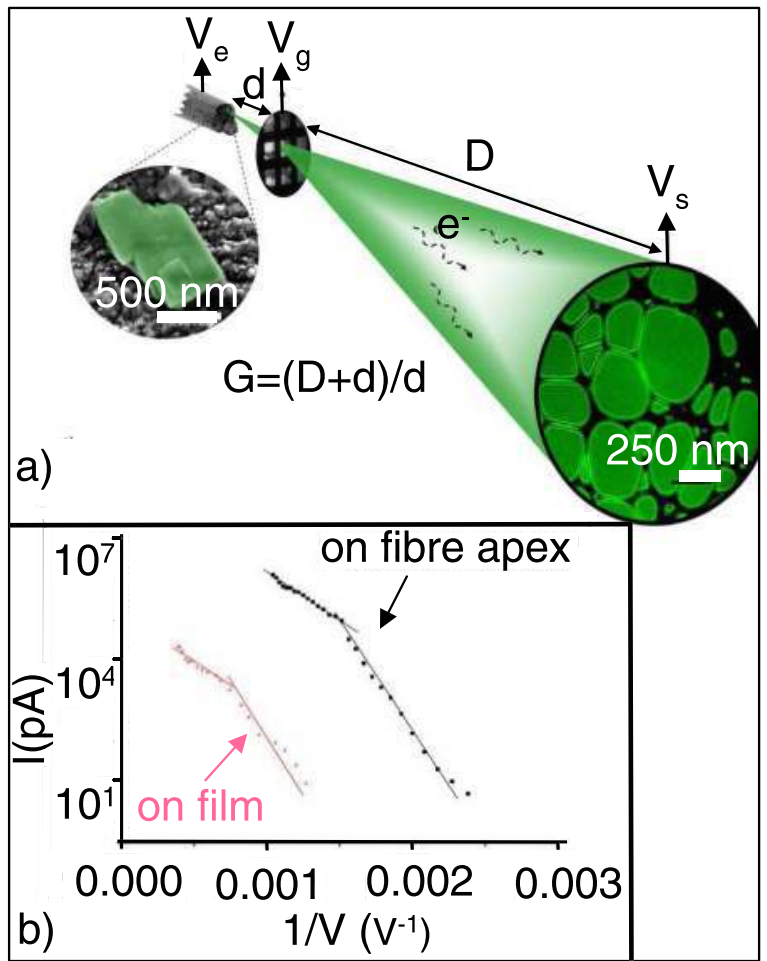

FIG. 3. (Color online) (a) Low energy electron projection microscopy using as source a single celadonite particle deposited at the apex of a $12 \mu \mathrm{m}$ diameter carbon fiber. The inset on the left shows an SEM image of the celadonite particle. (b) Fowler-Nordheim plot (black squares) for a celadonite particle deposited at the apex of a carbon fiber. For comparison, the $(+)$ plot is for a single celadonite particle deposited on a flat carbon surface.

(under)estimated by attributing measured current values to an area of $10 \times 10 \mathrm{~nm}^{2}$. The current is measured on the extraction grid, and corresponds to the current from one single particle deposited at the apex of the fiber. An I vs V characteristic for one celadonite particle is shown in Fig. 3(b).

Stability of the emission current is a crucial application parameter for any source, principally with respect to longterm stability in given vacuum conditions and amplitude and spectrum of current fluctuations around its average value. To study this stability, we record the current emitted by a fibertype source with a single celadonite particle at the apex. When recording is performed over a long period, the current is measured (every $0.1 \mathrm{~s}$ ) using a voltage data logger (NI USB-6009) via the current-to-voltage amplifier Keithley 427. These records show that emission lasts for months even at relatively high pressure, as high as $10^{-7}$ mbar. It lasts for hours for a $10^{-5}$ mbar pressure. The current fluctuations are huge at low current $(<100 \mathrm{nA})$ but we observe that current variations at higher current (approximately a few $\mu \mathrm{A}$ ) are greatly reduced [Fig. 4(a)]. Characterizing the fluctuations that occur at low current is useful to elucidate the emission mechanism: at a short time scale, the current is very stable, changing less than $1 \%$ for durations ranging from $0.1 \mathrm{~s}$ up to almost $1 \mathrm{~min}$. This behavior is not observed for higher current. To illustrate this behavior, the current for a constant emission voltage is plotted versus the time logarithm in Fig. 4(a), which shows short and long time scale behaviors, i.e., a discrete number of current values for the short time scale. A
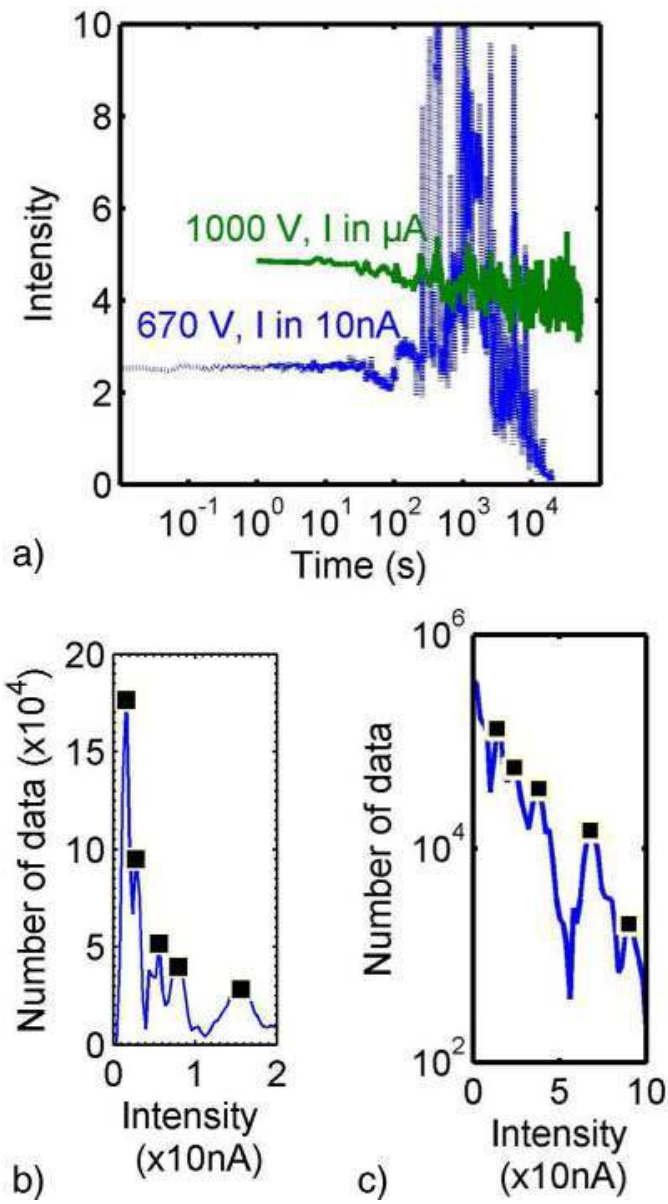

FIG. 4. (Color online) Emission intensity vs time for a constant emission voltage $\mathrm{V}_{\mathrm{e}}$ of a source consisting of a celadonite particle deposited on a fiber in front of an extractor grid. The current is measured on the extractor grid. The logarithmic scale for time compares variations occurring over short and long times. The solid line plot corresponds to $\mathrm{V}_{\mathrm{e}}=1000 \mathrm{~V}$, the current varies between 3.5 and $5.5 \mu \mathrm{A}$. The dashed line plot corresponds to $\mathrm{V}_{\mathrm{e}}=670 \mathrm{~V}$, the current varies between 2 and $80 \mathrm{nA}$.

histogram of intensity extracted from recording emission intensity over several days is shown in Figs. 4(b) and 4(c).

\section{Emission mechanism}

The I vs $\mathrm{V}$ curves at the single particle level, either on a film or on a fiber, are similar to those recorded for a large collection of mineral particles deposited on a film, ${ }^{21}$ i.e., a threshold field of a few V/ $\mu$ m [Fig. 2(c)], a Fowler-Nordheim regime at low current where the current strongly increases with voltage, and at higher current a regime where the current increases less strongly with voltage [Fig. 3(b)]. This supports the notion of a common underlying emission mechanism, which is easier to understand when approached from the single particle level. This approach is in fact quite similar to that taken for diamond film emission, where after the first observations on films, ${ }^{29}$ experiments on single diamond crystals were carried out to clarify the mechanism. ${ }^{30}$ Here, in addition to the microscopic images of mineral particles, the mechanism that we propose takes into account the main experimental facts, i.e., 
(1) Fowler-Nordheim behavior of I vs V characteristics at low current (and its deviation at higher current), suggesting a field emission mechanism

(2) a low macroscopic field (compared to local field emission measured in well defined metal experiments)

(3) relatively low sensitivity to high pressure, in contrast to field emission from metal tips

(4) discrete current values for emission at low current.

We start by considering a typical micrograph of a single particle of celadonite deposited on carbon (Fig. 1). Its lateral dimensions are micrometric while it is a few tens of nanometers thick (like other emitting minerals previously studied $\left.^{21}\right)$. The deposition of such a particle on a flat surface likely leads to only a few mechanical point contacts [Fig. $5(\mathrm{a})$. Subjected to a field of some $\mathrm{V} / \mu \mathrm{m}$, this particle positively charges, as already observed a long time ago for various insulators "subjected to high voltage stresses parallel to the dielectric surface."31,32 This involves, in our case, the edges of the particles (note that Refs. 29 and 30 also report emission from crystal edges). Charge density $\sigma$ so generated amounts to some $10^{5} \mathrm{C} / \mathrm{m}^{2}$, ${ }^{31}$ which creates fields $(\sigma / \varepsilon$ of the order of some $\mathrm{V} / \mu \mathrm{m}$. These fields are too weak to directly trigger field emission from carbon. But because of the applied field and the limited thickness of the particles, these positive charges migrate toward the negatively charged carbon substrate, and accumulate in front of any asperity A of the carbon surface [Fig. 5(b)] by electrostatic influence. Field emission from the asperity A occurs if the accumulation of positive charges is great enough so that the local field induced at $\mathrm{A}$ reaches conventional field emission values. The point is that only a small amount of positive charge is needed to trigger field emission from the substrate if $\mathrm{A}$ is close enough to the particle edge (a single elementary charge produces in vacuum a field of $0.36 \mathrm{~V} / \mathrm{nm}$ at a $2 \mathrm{~nm}$

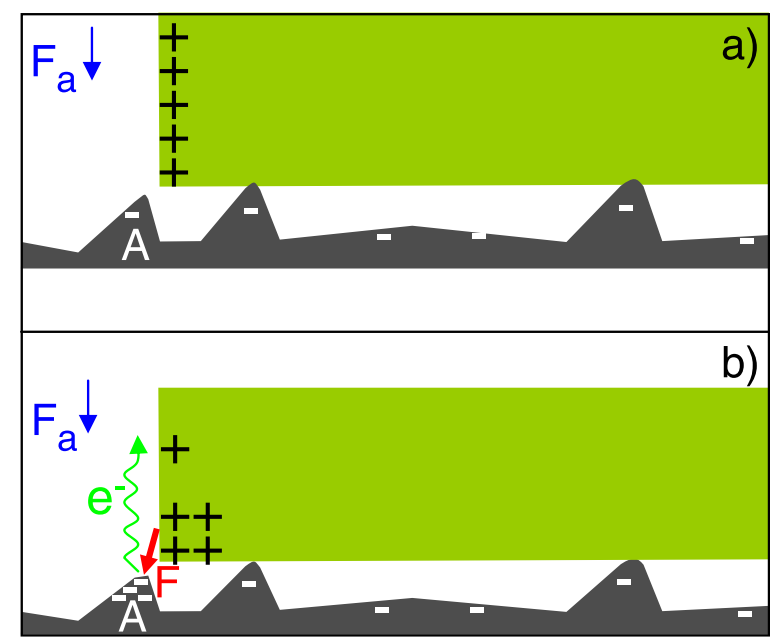

FIG. 5. (Color online) Theoretical model of the emission mechanism. The green plate is the celadonite particle. The gray support is the carbon substrate. $F_{a}$ is the applied field. A is an asperity close to a celadonite particle edge. (a) Positive charging of the lateral surface of the celadonite particle induced by the applied field. (b) Field induced migration of the positive charges toward the asperity $\mathrm{A}$ creates an intense field $\mathrm{F}$ localized at $\mathrm{A}$, and electron field emission from $\mathrm{A}$. distance). It has been shown ${ }^{31}$ that the charge density $\sigma$ along the edge of the insulators is proportional to the applied voltage. If the charge migration process is linear, at least in the applied voltage range considered here, the accumulation in front of $\mathrm{A}$ and therefore the local field at $\mathrm{A}$ is proportional to the applied field. The emission current I versus applied voltage $\mathrm{V}$ thus follows a Fowler-Nordheim dependence as shown in Fig. 3. It is expected that, at high current, part of the emission current is collected by the insulator, neutralizing positive charges and producing the deviation from Fowler-Nordheim behavior (Fig. 3). This description explains the first two experimental facts previously listed, as well as the third one, i.e., the relatively low sensitivity to high pressure: the gas atoms ionized by the electron beam are not focussed toward the electron source by the low applied field lines, thus preventing source sputtering and subsequent source instability.

The above description is also supported by an analysis of current values at low current where current plateaus as long as $1 \mathrm{~min}$ are observed. These plateaus are visible as peaks in the histograms [Figs. 4(b) and 4(c)] of the current values obtained by recording current for several hours at constant voltage.

In our model, the number $\mathrm{n}$ of positive charges accumulated in front of A governs the local field $F$ at A and therefore the emitted current

$$
I \approx \exp \left(-\beta \Phi^{3 / 2} / F\right)
$$

where $\beta$ is a slowly varying function of field, and $\Phi$ is the work function of carbon. The field $F$ is the sum of the contribution of each positive charge (and their negative induced charge near A). Because the macroscopic applied field is at least two orders of magnitude smaller than the local field, we expect in a first approximation that

$$
F \approx n f
$$

where the contribution of each charge $f$ is supposed to be a constant, therefore

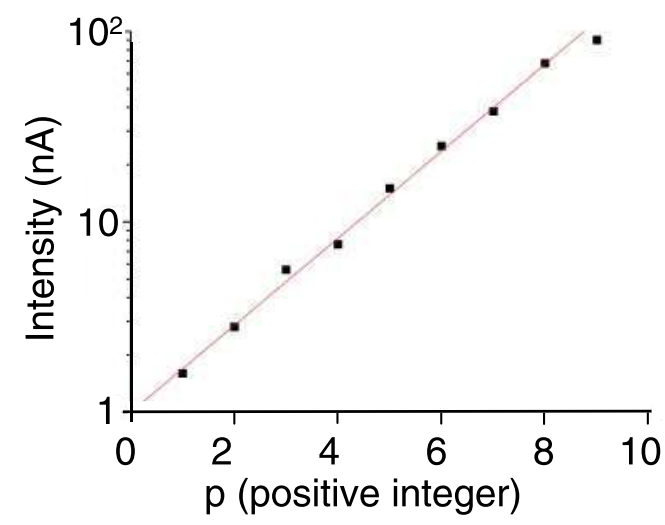

FIG. 6. (Color online) Logarithm of the most frequent values of the emission current intensity I vs p, a positive integer related to the number of charges responsible for the field emission current. The current is measured on the extractor grid where $\mathrm{V}_{\mathrm{g}}=0 \mathrm{~V}$ and the voltage at the emitter is fixed at $\mathrm{V}_{\mathrm{e}}=670 \mathrm{~V}$ for several hours. 


$$
I \approx \exp \left[-\beta \Phi^{3 / 2} /(n f)\right],
$$

changes in $n$ around its average value $n_{0}$ induce current changes

$$
\ln I \approx-\beta \Phi^{3 / 2} /\left(n_{0} f\right)+\beta\left(n-n_{0}\right) \Phi^{3 / 2} /\left(n_{0}^{2} f\right) .
$$

Identifying the most frequent current values [corresponding to the maxima of the histogram of Figs. 4(b) and 4(c)] with values of well-defined $n$ means that the logarithms of these most frequent values are proportional to an integer

$$
p=n-n_{0} .
$$

Confirmation of this behavior is shown in Fig. 6. Note that the absolute number of charges is not known but is small if the distance between A and the particle edge is small. At high current the current plateaus are not observed. We suggest that unequal contributions to the field from the individual charges, as a result of the large number $n$ of charges involved, smears the discreteness of current values.

\section{SUMMARY AND CONCLUSIONS}

In conclusion, we show that at a field as low as a few $\mathrm{V} / \mu \mathrm{m}$ electron field emission occurs from a single mineral particle deposited on carbon. Based on the previous observations of positive charges (the origin of which is presently not known) for such low fields applied on macroscopic insulators by De Tourreil et al. ${ }^{31}$ and because of the nanometer thickness of mineral particles, we suggest that nanometer scale migration of a small number of these charges could lead to high field on an asperity of the carbon support close to the edge of the particle, and triggers conventional field emission. At low current, the fluctuations of the emission current would thus appear to be related to the fluctuations of the number of positive charges accumulated on the particle as supported by an analysis of the observed fluctuations. Electron point sources based on this emission process are bright and able to work for days in relatively poor vacuum conditions compared to metal field emission tips.

One referee of the present paper points to us a method ${ }^{33}$ based on Millikan-Lauritsen plots (log I vs $1 / \mathrm{V})$ analysis (Fig. 3 of this manuscript) and gives estimates of the local field in our experiments: 3.2 to $4.7 \mathrm{~V} / \mathrm{nm}$ for the low voltage straight-line section of Fig. 3 assuming a work function of $5.1 \mathrm{eV}$. Estimates of the field at the apex of the fiber is about $\mathrm{V} /(5 \mathrm{r}),{ }^{14}$ where $\mathrm{r}$ is the fiber radius $(6 \mu \mathrm{m})$ for a turn-on macroscopic field of $14 \mathrm{~V} / \mu \mathrm{m}(\mathrm{V}=420 \mathrm{~V})$. Although this value is five times the turn-on macroscopic field observed on film, the value on the fiber might be overestimated since the flatness of the fiber apex.

\section{ACKNOWLEDGMENT}

The authors thank Marjorie Sweetko for English language revision.

${ }^{1}$ R. G. Forbes, Solid State Electron. 45, 779 (2001).

${ }^{2}$ N. S. Xu, Y. Tzeng, and R. V. Latham, J. Phys. D: Appl. Phys. 26, 1776 (1993).

${ }^{3}$ O. Groning, O. M. Kuttel, P. Groning, and L. Schlapbach, J. Vac. Sci. Technol. B 17, 1970 (1999).

${ }^{4}$ A. N. Banerjee and S. W. Joo, Nanotechnology 22, 365705 (2011).

${ }^{5}$ G. Eda, H. E. Unalan, N. Rupesinghe, G. A. J. Amaratunga, and M. Chhowalla, Appl. Phys. Lett. 93, 233502 (2008).

${ }^{6}$ D. Banerjee, S. H. Jo, and Z. F. Ren, Adv. Mater. 16, 2028 (2004).

${ }^{7}$ W. Zhu, G. P. Kochanski, and S. Jin, Science 282, 1471 (1998).

${ }^{8}$ M. W. Geis, J. C. Twichell, and T. M. Lyszczarz, J. Vac. Sci. Technol. B 14, 2060 (1996).

${ }^{9}$ I. Alexandrou, E. Kymakis, and G. A. J. Amaratunga, Appl. Phys. Lett. 80, 1435 (2002).

${ }^{10}$ S. A. Furkert, A. Wotherspoon, D. Chems, N. A. Fox, G. M. Fuge, P. J. Heard, and S. P. Lansley, Appl. Phys. Lett. 90, 242109 (2007).

${ }^{11}$ M. S. Chung, S. C. Hong, P. H. Cutler, N. M. Miskovsky, B. L. Weiss, and A. Mayer, J. Vac. Sci. Technol. B 24, 909 (2006).

${ }^{12}$ S. Sun and L. K. Ang, J. Appl. Phys. 112, 066102 (2012).

${ }^{13}$ R. Morin, Surf. Sci. 162, 109 (1985).

${ }^{14}$ R. Gomer, Field Emission and Field Ionization (Harvard University, Cambridge, MA, 1961).

${ }^{15}$ K. L. Jensen, J. Vac. Sci. Technol. B 21, 1528 (2003).

${ }^{16}$ I. S. Altman, P. V. Pikhitsa, and M. Choi, Appl. Phys. Lett. 84, 1126 (2004).

${ }^{17}$ G. S. Gipson and H. C. Eaton, J. Appl. Phys. 51, 5537 (1980).

${ }^{18}$ N. S. Xu and S. E. Huq, Mater. Sci. Eng. R 48, 47 (2005).

${ }^{19}$ V. V. Zhirnov, G. J. Wojak, W. B. Choi, J. J. Cuomo, and J. J. Hren, J. Vac. Sci. Technol. A 15, 1733 (1997).

${ }^{20}$ K. H. Bayliss and R. V. Latham, Proc. R. Soc. London, Ser. A 403, 285 (1986).

${ }^{21}$ J. Rech, O. Grauby, and R. Morin, J. Vac. Sci. Technol. B 20, 5 (2002).

${ }^{22}$ R. Daineche, A. Degiovanni, O. Grauby, and R. Morin, Appl. Phys. Lett. 88, 023101 (2006).

${ }^{23}$ A. T. Davidson and A. F. Vickers, J. Phys. C Solid State 5, 879 (1972).

${ }^{24}$ F. Pesty and P. Garoche, Surf. Sci. 580, 153 (2005).

${ }^{25}$ V. Drahos and A. Delong, Nature 186, 104 (1960).

${ }^{26}$ R. Morin, A. Gargani, and F. Bel, Microsc. Microanal., Microstruct. 1, 289 (1990).

${ }^{27}$ H. W. Fink, W. Stocker, and H. Schmid, Phys. Rev. Lett. 65, 1204 (1990).

${ }^{28}$ A. Beyer and A. Goelzhaeuser, J. Phys.: Condens. Matter 22, 343001 (2010).

${ }^{29}$ C. Wang, A. Garcia, D. C. Ingram, M. Lake, and M. E. Kordesch, Electron. Lett. 27, 1459 (1991).

${ }^{30}$ M. W. Geis, N. N. Efremow, K. E. Krohn, J. C. Twichell, T. M. Lyszczarz, R. Kalish, J. A. Greer, and M. D. Tabat, Nature 393, 431 (1998).

${ }^{31}$ C. H. De Tourreil, K. D. Srivastava, and U. J. Woelke, IEEE Trans. Electr. Insul. E1-7, 176 (1972).

${ }^{32}$ C. H. De Tourreil and K. D. Srivastava, IEEE Trans. Electr. Insul. E1-8, 17 (1973).

${ }^{33}$ R. G. Forbes, Proc. R. Soc. A 469, 0271 (2013). 